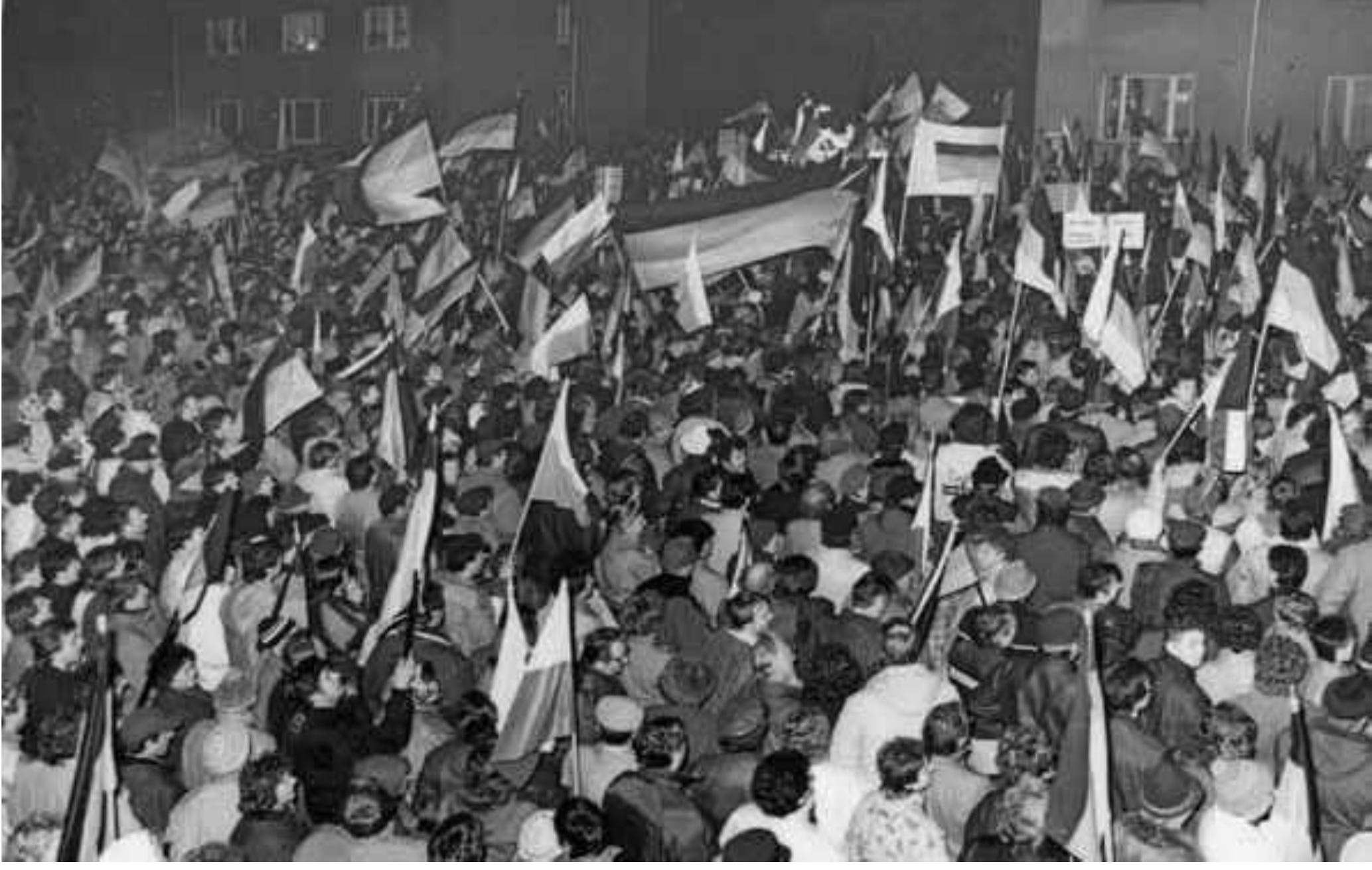

\title{
Rückblicke auf die Friedliche Revolution in Limbach-Oberfrohna
}

\author{
Reinhard Schnabl
}

Friedensgebete, Demonstrationen, Runder Tisch - in dieser Reihenfolge fanden 1989 in LimbachOberfrohna, wie in vielen Städten unseres Landes, Willensbekundungen der Menschen statt, die nicht mehr wegsehen wollten, was in ihrem Lande geschah. Auch wenn die Genossen immer noch sangen: „Die Partei hat immer recht...“, glaubte ihnen das Volk nicht mehr. So wollten viele einen Neubeginn. Es war nur die Frage, wie man diesem Willen die Möglichkeit geben konnte, sich zu artikulieren. Darüber machten sich auch in unserer Stadt verantwortungsvolle Bürger Gedanken, welche sich später im „Neuen Forum" engagierten, und die Limbacher Pfarrerschaft, welche von Gemeindegliedern in ihrer Not angesprochen wurden.

Das Ergebnis waren Friedensgebete in den Kirchen der Stadt. Hier versammelten sich von Woche $\mathrm{zu}$ Woche immer mehr Menschen in den Kirchen. Ich hatte den Eindruck, die Menschen suchten und fanden hier einen Schutzraum; sie erwarteten von uns Pfarrern wegweisende Worte und von Gott, dass er unsere Gebete erhört. Darin waren sich damals Christen und Nichtchristen sehr ähnlich. Im Nachhinein wissen wir: Es war gut, dass wir Christen damals unsere Kirchentüren öffneten und nicht mehr unter uns blieben. Vierzig Jahre hatten wir Schutz gesucht und gefunden hinter dicken Kirchenmauern, einen Freiraum in unseren Pfarrhäusern den Menschen geboten. Zwar mussten wir immer befürchten, dass einer mithört und darüber berichtet, was in der Kirche geredet wird, aber der Glaube und die Gemeinschaft gaben vielen immer wieder den Mut, frei zu reden. So durfte ich erleben, dass Eltern ihre Kinder konfirmieren ließen, auch wenn sie von der Schule bedrängt wurden. Oder dass junge Männer zu den Bausoldaten gingen oder total den Wehrdienst verweigerten und dafür ins Gefängnis mussten. Als Kirche erinnerten wir an die Schöpfungsverantwortung des Menschen, gerade in der um-
Demonstration auf dem Johannisplatz in LimbachOberfrohna im November 1989 ( ) Stadtarchiv Limbach-Oberfrohna 
weltzerstörenden Planwirtschaft der DDR. Ich selbst trug als Pfarrer den Aufkleber „Schwerter zu Pflugscharen" auf meiner Jacke gegen die maßlose Aufrüstung in Ost und West. Ich denke, dies blieb bei Christen und Nichtchristen nicht unbemerkt. Im Herbst 1989 war dann die Stunde des Protestes gekommen. Wir waren uns einig: der Protest sollte friedlich sein. Und so kamen die Menschen in unsere Kirchen und hörten geduldig und begierig die Auslegung des Evangeliums mit direktem Zeitbezug. Wenn die Menschen nach den Friedensgebeten aus den Kirchen auf die Straße strömten, waren auch meine Frau und ich dabei. Wir hatten zuvor der Oma gesagt: „Pass auf die Kinder auf. Wenn wir nicht wiederkommen, du weißt, wo wir sind“. Unsere Ausrüstung bestand immer aus zwei Kerzen, die wir in der Hand trugen. Das war bei allen so, die durch Limbach zogen, hin zum Johannisplatz, zur Kellerwiese, zur Kundgebung.

Und das Wunder geschah, dass alles friedlich blieb. Ja, die Polizei schützte den Demonstrationszug, und die Menschen vergaßen, ihre Fäuste zu ballen. Die Gewalt blieb aus, auch wenn die Rufe der Demonstranten zunehmend aggressiver wurden. Auf der Kundgebung hörten dann alle Zeugnisse der Betroffenheit, die alle bewegten. Hier sprachen Menschen, die Unrecht erduldet hatten und bisher nicht $\mathrm{zu}$ reden wagten. Nun brach es aus ihnen heraus, die Bespitzelung, die Gängelung und Bevormundung durch den Staat. So wurde in den Demos der Ruf laut: „Stasi in die Produktion“, bis das Bewusstsein der eigenen Stärke in der Parole mündete: „Wir sind das Volk“. Als die Staatsführung in den kommenden
Wochen und Monaten in Agonie verharrte, unfähig für Veränderungen, kamen neue Rufe auf: „Wir sind ein Volk“ oder „Wenn die DM nicht zu uns kommt, kommen wir zu ihr".

Am 1. November 1989 gingen in LimbachOberfrohna nach einem Friedensgottesdienst in der Oberfrohnaer Lutherkirche 8.000 Menschen auf die Straße. Im Limbacher Rathaushof fand anschließend die erste öffentliche Kundgebung statt, bei der die Bürgerinitiative Limbach-Oberfrohna und das Neue Forum auftraten. Am 8. November 1989 beteiligten sich rund 20.000 Menschen an der Kundgebung - das waren mehr Demonstrationsteilnehmer, als Limbach-Oberfrohna damals Einwohner hatte! Auch die nachfolgenden Mittwochs-Demonstrationen hatten erstaunlichen Zulauf.

Nach dem Mauerfall nahm die Geschwindigkeit der Veränderungen im Volk rasant zu. In dieser Zeit wurde der Runde Tisch in Limbach-Oberfrohna gegründet. Vertreter der Bürgerinitiative, von Parteien und der Kirche trafen sich hier über mehrere Monate wöchentlich, um die Sorgen und Nöte der Bürger ins Rathaus zu tragen und deren Forderungen Nachdruck zu verleihen. Ich möchte nur einige Tagesordnungspunkte nennen, die uns damals beschäftigten:

- Entzug der Standortgenehmigung für den VEB Polychemie in Oberfrohna wegen extremer Umweltbelastung

- Überführung des SED-Gästehauses in Kändler in Volkseigentum

- Investorensuche für Betreiber der „Parkschänke“
Bericht in der "Freien Presse" vom 15. November 1989

\section{In Limbach-Oberfrohne demonstrierten am Mitwochabend 20000 Menschen $15,11 . \mathrm{ps}$}

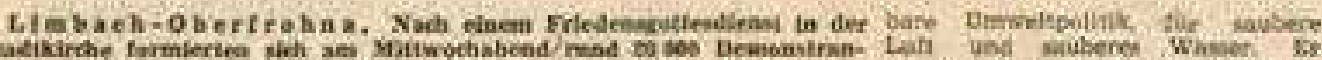

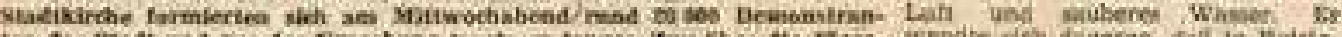

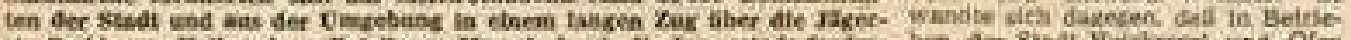

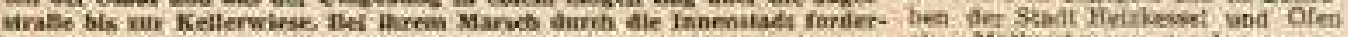

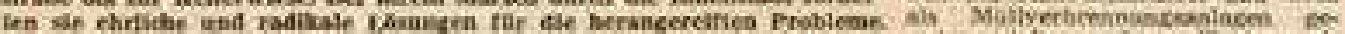

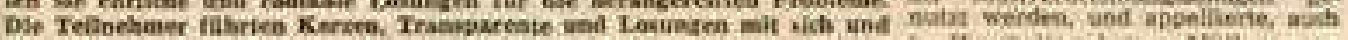

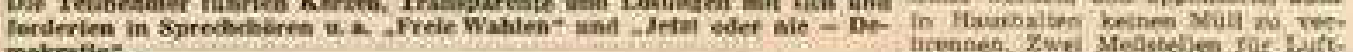
mokratie:s.

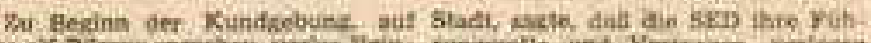

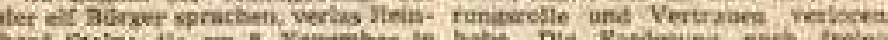

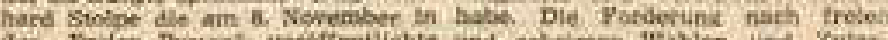

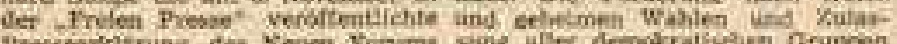

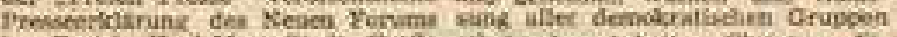

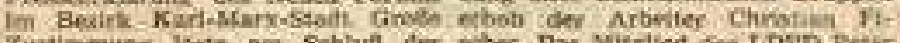

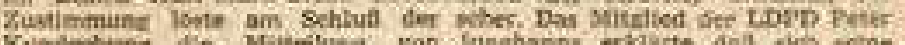

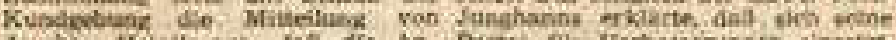

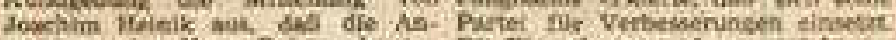

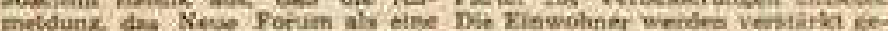

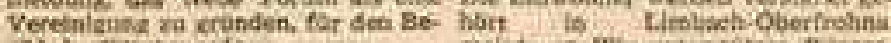

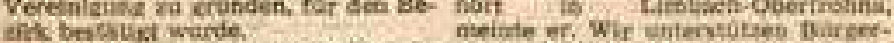

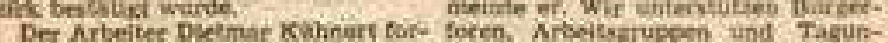

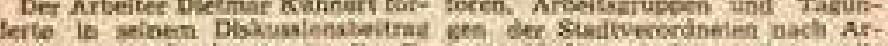

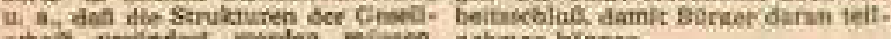

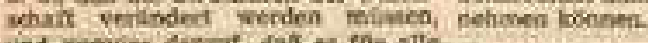

und remins langif, dab en ror alle

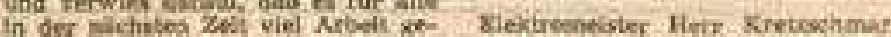

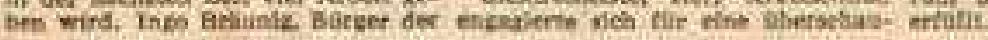
werle wibes in der stadi einierieb: iet werden.

Yriedmar, schiahmann, viele Jahre Eetrieledicekion In YEB Brecta: chatisulk und yetrt Invalisennto:-

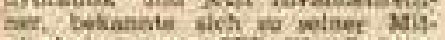
atiedschan is ter stab. Mut-ntuce et. conies. Aler toh iteile mech - brave luswo. riefen Writ = und lece nuch jetrt die Hainde nicht in den Schis.

Dermonatration und Kuidisebone verlielen teledisch, weweitireb und

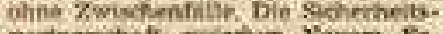
partietichafs zwistiva kecem Fo- 
- Beräumen eines Waffenlagers im Rathaus

- Umsetzung der sogenannten „Modrowgesetze“, welche Eigenheimbesitzern noch vor der deutschen Einheit einen Kauf ihres Baulandes ermöglichten und

- nicht zuletzt die Vorbereitung der ersten freien Kommunalwahlen in Limbach-Oberfrohna seit 1933

Die Kommunalwahlen fanden am 6. Mai 1990 statt. Der Runde Tisch hatte seine Schuldigkeit getan. Wir wussten alle, es war ein Interim, ein Übergang zu einer freiheitlichen, von den Bürgern gewählten Vertretung. Zwei Dinge sind mir in dieser Zeit aufgefallen. Das Interesse an diesen Wahlen war in der Bevölkerung riesig. Auch aus den Limbach-Oberfrohnaer Kirchgemeinden kandidierten viele Gemeindeglieder für den Stadtrat oder stellten sich als Wahlhelfer zur Verfügung. Ich erinnere mich auch, dass 1990 Wahlen zum Kirchenvorstand stattfanden und ich Mühe hatte, Kandidaten zu finden, weil sich viele Gemeindeglieder bereits politisch engagiert hatten. Ich erinnere mich auch an die vielen Fahnen, die in dieser Zeit wehten, Deutschlandfahnen ebenso wie Sachsenfahnen.

13.837 wahlberechtigte Bürger von LimbachOberfrohna nahmen ihr Wahlrecht wahr. Das Ergebnis war sehr deutlich. Die CDU erhielt in Limbach-Oberfrohna die meisten Stimmen, gefolgt von der SPD und der Deutschen Forumpartei, einer Abspaltung des Neuen Forums, die sich dem liberalen Wahlbündnis angeschlossen hatte. Hier wirkte sicherlich der Kanzlerbonus nach. Helmut Kohl trauten damals die Menschen am ehesten die Verwirklichung der Deutschen Einheit und die Lösung ihrer Probleme zu. So bekam die CDU auch in Limbach-Oberfrohna einen Vertrauensvorschuss für die Neugestaltung unserer Stadt. Am 29. Mai 1990 fand die konstituierende Sitzung des neuen Stadtrates statt. Nach einem ökumenischen Gottesdienst begaben sich alle in das Kulturhaus „Rudolf Marek“, wo auf der ersten Sitzung des neuen Stadtrates, welche ich als Vertreter der Kirche eröffnen durfte, personell die Weichen zukünftiger Arbeit des Stadtrates gestellt wurden.

Nachdem die D-Mark eingeführt und die Deutsche Einheit vollendet worden war, erlebten wir alle in einem rasanten Tempo, wie die Marktwirtschaft der alten Bundesländer die Planwirtschaft des Ostens binnen kurzem platt machte. Nach der Mangelwirtschaft vergangener Tage erlebten wir nun den Überfluss. Nach der Diktatur des Proletariats waren wir plötzlich in der Demokratie gelandet. Wie man sich darin bewegen kann und soll, mussten wir möglichst sofort lernen. Unsere Brüder und Schwestern in den alten Bundesländern hatten dafür vierzig Jahre Zeit. Erstaunlich, wie schnell wir gelernt haben. Landwirte fingen wieder an, ihr eigenes Land zu bewirtschaften, Arbeiter und Angestellte wurden selbständig, andere schulten um, um neue Arbeit $\mathrm{zu}$ finden, mache verließen auch ihre Heimat und gingen dorthin, wo es Arbeit gab. Viele Menschen erfüllten sich eine lang gehegte Sehnsucht - zu reisen, wohin sie wollten, privat oder mit der Gemeinde. So gab es in den ersten zehn Jahren nach der Wende regelmäßige Besuche in den Partnerkirchgemeinden Mittegroßefehn in Ostfriesland und Dachau bei München. Endlich konnten wir deren Besuche bei uns erwidern. Der Kirchenvorstand unternahm in dieser Zeit zwei Reisen nach Ungarn, welche nicht nur unseren Horizont erweiterten, sondern auch die Gemeinschaft stärkten.

So durfte ich als Pfarrer an vielen positiven Entwicklungen teilhaben und habe deshalb ein Gefühl der Dankbarkeit, wenn ich an die letzten 25 Jahre zurückdenke. Ich bin auch dankbar, dass ich zehn Jahre Pfarrer in der DDR war, weil ich dadurch vergleichen kann, wie es damals war und heute ist. Nicht alles ist besser geworden $\mathrm{zu}$ damals. Nostalgie kommt bei mir dennoch nicht auf, da ich die ideologische Bevormundung des kommunistischen Systems und die daraus erwachsene Unfreiheit des Einzelnen nicht vergessen habe. Mein Wunsch wäre, dass jeder einzelne Bürger und besonders die in der Politik Verantwortlichen dieses hohe Gut der Freiheit des Einzelnen pflegen und fördern. „Suchet der Stadt Bestes“ ist im Stadtwappen von LimbachOberfrohna zu lesen und ich möchte den Satz vollenden, wie er in der Bibel steht: „denn geht es ihr gut, so geht es auch euch gut".

2009 erklärte der Stadtrat von Limbach-Oberfrohna in Erinnerung an den Beginn der öffentlichen Proteste im Herbst 1989 den 1. November zum Gedenktag an die Friedliche Revolution. Außerdem wurde im Rathaushof, wo mehrere Kundgebungen stattgefunden hatten, eine Stele zur Erinnerung an die Demonstrationen in Limbach-Oberfrohna errichtet. Sie ist mit Losungen des Jahres 1989 gestaltet.

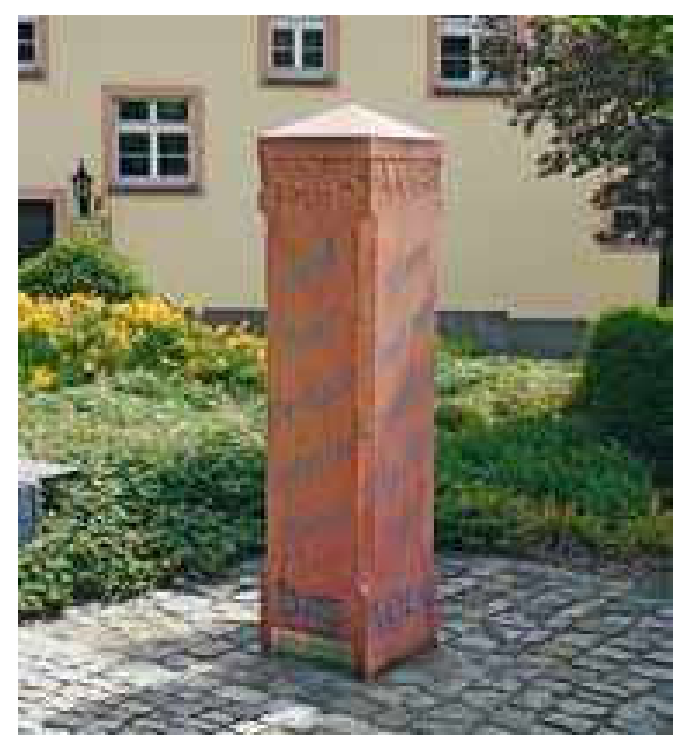

Stele zum Gedenken an die Friedliche Revolution in Limbach-Oberfrohna Foto: Matthias Donath

\section{Autor}

Pfarrer i. R. Reinhard Schnabl 1980-2015 Pfarrer in Rußdorf, 2003-2015 auch in Bräunsdorf 